\title{
ALMOST ADDITIVE-QUADRATIC-CUBIC MAPPINGS IN MODULAR SPACES
}

\author{
MOHAMMAD MAGHSOUDI, ABASALT BODAGHI, ABOLFAZL NIAZI MOTLAGH, \\ AND MAJID KARAMI
}

\begin{abstract}
We introduce and obtain the general solution of a class of generalized mixed additive, quadratic and cubic functional equations. We investigate the stability of such modified functional equations in the modular space $X_{\rho}$ by applying the $\Delta_{2}$-condition and the Fatou property (in some results) on the modular function $\rho$. Furthermore, a counterexample for the even case (quadratic mapping) is presented.
\end{abstract}

\section{INTRODUCTION}

In 1940, Ulam raised the first stability problem before a Mathematical Colloquium at the University of Wisconsin, where he presented a list of unsolved problems that were published years later in the book [38. He posed the question of whether there exists an exact homomorphism near an approximate homomorphism. An answer to the problem was partially given by Hyers [12 for linear functional equations in the setting of Banach spaces. Later on, various generalizations and extension of Hyers' result were ascertained by Aoki 1 for additive mappings, Th. M. Rassias [34] for linear mappings by considering an unbounded Cauchy difference, and also J. M. Rassias [32, Gajda [11] in different versions. Over the last seven decades, the Ulam problem was tackled by numerous authors, and its solutions via miscellaneous forms of functional equations like additive [37, quadratic [36, 8], cubic [18, 26], quartic [3, 20], and mixed type functional equations [4, 6, 9, 28, 41] which involve only these types of functional equations were discussed.

The functional equation $f(x+y)+f(x-y)=2 f(x)+2 f(y)$ is related to symmetric bi-additive functions. Since $f(x)=c x^{2}$ is its solution, it is natural that this equation is called a quadratic functional equation. In particular, every solution of the quadratic equation is said to be a quadratic mapping.

In 2001, J. M. Rassias [33] introduced the cubic functional equation

$$
f(x+2 y)-3 f(x+y)+3 f(x)-f(x-y)=6 f(y)
$$

2010 Mathematics Subject Classification. 39B72, 39B82, 47H09.

Key words and phrases. Additive-quadratic-cubic functional equation; Modular spaces; HyersUlam stability. 
and established the solution of the Hyers-Ulam stability problem for these cubic mappings. It is easy to see that the function $f(x)=a x^{3}$ satisfies 1.1 . The alternative cubic functional equation

$$
f(2 x+y)+f(2 x-y)=2 f(x+y)+2 f(x-y)+12 f(x)
$$

has been introduced by Jun and Kim in [13. They found out the general solution and established the Hyers-Ulam stability for the functional equation 1.2 . Moreover, in [14] they introduced the cubic functional equation

$$
f(x+2 y)+f(x-2 y)=4 f(x+y)+4 f(x-y)-6 f(x)
$$

and investigated the Hyers-Ulam stability problem for it. In [7], Bodaghi et al. introduced a generalization of the cubic functional equation as follows:

$$
\begin{aligned}
f(x+n y)+ & f(x-n y)=2\left(2 \cos \left(\frac{n \pi}{2}\right)+n^{2}-1\right) f(x) \\
& -\frac{1}{2}\left(\cos \left(\frac{n \pi}{2}\right)+n^{2}-1\right) f(2 x)+n^{2}\{f(x+y)+f(x-y)\}
\end{aligned}
$$

for an integer $n \geq 1$. They determined the general solution and proved the HyersUlam stability problem for the functional equation (1.3) related to cubic Jordan *-derivations. The stability of the equation $[1.3$ in various spaces is studied in [5]. The cubic functional equation which is the generalization form of the mentioned versions was given in 2 as follows:

$$
f(m x+n y)+f(m x-n y)=m n^{2}\{f(x+y)+f(x-y)\}+2 m\left(m^{2}-n^{2}\right) f(x),
$$

where $m, n$ are integer numbers with $m \geq 2$.

Nakano [27] initiated the study of modulars on linear spaces and the related theory of modular linear spaces as generalizations of metric spaces. After that, Luxemburg [21, Mazur, Musielak and Orlicz [23, 24, 25] thoroughly developed it extensively. Since then, the theory of modulars and modular spaces has been widely applied in the study of interpolation theory [22, 19] and various Orlicz spaces [29]. A modular yields less properties than a norm does, but it makes more sense in many special situations. When we work in a modular space, it is frequently assumed that the modular satisfies extra additional properties like some relaxed continuity or some $\Delta_{2}$-condition. As for the mentioned condition, Khamsi [15] studied the stability of quasicontraction mappings in modular spaces without $\Delta_{2}$-condition by using the fixed point theorem. The stability results of additive functional equations in modular spaces equipped with the Fatou property and $\Delta_{2}$ condition were investigated by Sadeghi [35], who used Khamsi's fixed point theorem. In addition, the stability of quadratic functional equations in modular spaces satisfying the Fatou property without using the $\Delta_{2}$-condition was investigated in [40]. Recently, Park, Bodaghi, and Kim [30] investigated the stability of additive and Jensen-additive functional equations without using the $\Delta_{2}$-condition by a fixed point method. An alternative generalized Hyers-Ulam stability theorem of a modified quadratic functional equation in a modular space using the $\Delta_{3}$-condition without the Fatou property on a modular function can be found in [16]. In addition, a refined stability result and alternative stability results for additive and 
quadratic functional equations using the direct way (Hyers' method) in modular spaces are given in [17].

In [39], Wang et al. obtained the general solution and investigated the Ulam stability problem for the following mixed additive, quadratic and cubic functional equation

$$
\begin{aligned}
f(2 x+y)+f(2 x-y)= & 2\{f(x+y)+f(x-y)\} \\
& +2 f(2 x)-4 f(x)-f(y)-f(-y)
\end{aligned}
$$

in Banach spaces. A different form of a mixed additive, quadratic and cubic functional equation which is introduced in [10] is as follows:

$$
f(x+m y)+f(x-m y)=m^{2}\{f(x+y)+f(x-y)\}+2\left(1-m^{2}\right) f(x),
$$

where $m$ is a fixed integer with $m \neq 0, \pm 1$.

In this article, for two vector spaces $X$ and $Y$, we consider the mapping $f: X \rightarrow Y$ which is a common solution of the functional equations

$$
\begin{aligned}
f(m x+ & n y)+f(m x-n y)+f(n x+m y)+f(n x-m y) \\
= & m n(m+n)[f(x+y)+f(x-y)-2 f(x)-f(y)-f(-y)] \\
& +2[f(m x)+f(n x)]+\left(m^{2}+n^{2}\right)[f(y)+f(-y)]
\end{aligned}
$$

for the fixed integer $n$ and any integer $m$ such that $m, n \neq 0, \pm 1$ and $m+n \neq 0$. We note that each element of this class of functional equations can be considered as a combination of 1.4 and 1.5 in a general form. It is easily verified that the function $f(x)=\alpha x^{3}+\beta x^{2}+\gamma x$ is a solution of the functional equation 1.6 for all $m, n$ with $m, n \neq 0, \pm 1$ and $m+n \neq 0$. We obtain the general solution and study the Hyers-Ulam-Rassias stability of the equation 1.6 in the setting of modular spaces.

\section{Preliminary notations}

In this section, we recall some basic facts concerning modular spaces and some preliminary results.

Definition 2.1. Let $X$ be a linear space over a field $\mathbb{K}(\mathbb{R}$ or $\mathbb{C})$. A generalized function $\rho: X \rightarrow[0, \infty]$ is called a modular if it satisfies the following three conditions for elements $\alpha, \beta \in \mathbb{K}, x, y \in X$ :

(i) $\rho(x)=0$ if and only if $x=0$;

(ii) $\rho(\alpha x)=\rho(x)$ for all scalars $\alpha$ with $|\alpha|=1$;

(iii) $\rho(\alpha x+\beta y) \leq \rho(x)+\rho(y)$ for all scalars $\alpha, \beta \geq 0$ with $\alpha+\beta=1$.

If the condition (iii) is replaced by $\rho(\alpha x+\beta y) \leq \alpha^{t} \rho(x)+\beta^{t} \rho(y)$ when $\alpha^{t}+\beta^{t}=1$ and $\alpha, \beta \geq 0$ with a $t \in(0,1]$, then $\rho$ is called a $t$-convex modular. 1-convex modulars are called convex modulars. For a modular $\rho$, there corresponds a linear subspace $X_{\rho}$ of $X$, given by $X_{\rho}:=\{x \in X: \rho(\lambda x) \rightarrow 0$ as $\lambda \rightarrow 0\}$. In this case $X_{\rho}$ is called a modular space.

Let $\rho$ be a convex modular. Then, the modular space $X_{\rho}$ can be equipped with a norm called the Luxemburg norm, defined by $\|x\|_{\rho}=\inf \left\{\lambda>0: \rho\left(\frac{x}{\lambda}\right) \leq 1\right\}$.

We remind the following notes, which are taken from [16]: 
(1) If $\rho$ is a modular on $X$, then $\rho(t x)$ is an increasing function in $t \geq 0$ for each fixed $x \in X$, that is, $\rho(a x) \leq \rho(b x)$ whenever $0 \leq a<b$;

(2) If $\rho$ is a convex modular on $X$ and $|\alpha| \leq 1$, then $\rho(a x) \leq|\alpha| \rho(x)$ for all $x \in X$. In particular, if $\alpha_{j} \geq 0(j=1,2, \ldots, n)$ with $0<\sum_{j=1}^{n} \alpha_{j} \leq 1$, then $\rho\left(\sum_{j=1}^{n} \alpha_{j} x_{j}\right) \leq \sum_{j=1}^{n} \alpha_{j} \rho\left(x_{j}\right)$ for all $x_{j} \in X$.

Definition 2.2. Let $X_{\rho}$ be a modular space and let $\left\{x_{n}\right\}$ be a sequence in $X_{\rho}$. Then

(i) $\left\{x_{n}\right\}$ is $\rho$-convergent to a point $x_{*} \in X_{\rho}$ and we write $x_{n} \stackrel{\rho}{\rightarrow} x_{*}$ if $\rho\left(x_{n}-\right.$ $\left.x_{*}\right) \rightarrow 0$ as $n \rightarrow \infty$

(ii) $\left\{x_{n}\right\}$ is a $\rho$-Cauchy sequence if for any $\epsilon>0$ one has $\rho\left(x_{n}-x_{m}\right)<\epsilon$ for sufficiently large $m, n \in \mathbb{N}$;

(iii) A subset $Y \subseteq X_{\rho}$ is called $\rho$-complete if any $\rho$-Cauchy sequence is $\rho$ convergent to a point in $Y$.

Example $2.3([31])$. Let $\psi:[0, \infty) \rightarrow \mathbb{R}$ be a function defined by $\psi(0)=0$ and $\psi(t)>0$ for all $t>0$, and $\lim _{t \rightarrow \infty} \psi(t)=\infty$. If moreover $\psi$ is convex, continuous and nondecreasing, then $\psi$ is called an Orlicz function. For a measure space $\left(X, \sum, \mu\right)$, suppose that $L^{0}(\mu)$ is the set of all measurable functions on $X$. For each $f \in L^{0}(\mu)$, define $\rho_{\psi}(f)=\int_{X} \psi(|f|) d \mu$. Then, $\rho_{\psi}$ is a modular and the corresponding modular space is called an Orlicz space and denoted by

$$
L_{\psi}=\left\{f \in L^{0}(\mu): \rho_{\psi}(\lambda f) \rightarrow 0 \text { as } \lambda \rightarrow 0\right\} .
$$

One can check that $L_{\psi}$ is $\rho_{\psi}$-complete.

A modular function $\rho$ is said to satisfy the $\Delta_{s}$-condition if there exists $\kappa>0$ such that $\rho(s x) \leq \kappa \rho(x)$ for all $x \in X_{\rho}$. Throughout this paper, we say that the constant $\kappa$ is a $\Delta_{s}$-constant related to the $\Delta_{s}$-condition. Suppose that $\rho$ is convex and satisfies the $\Delta_{s}$-condition with $\Delta_{s}$-constant $\kappa$. If $\kappa<s$, then $\rho(x) \leq \kappa \rho\left(\frac{x}{s}\right) \leq$ $\frac{\kappa}{s} \rho(x)$, which implies $\rho=0$. Hence, we must have the $\Delta_{s}$-constant $\kappa \geq s$ if $\rho$ is convex modular. It is said that the modular $\rho$ has the Fatou property if and only if $\rho(x) \leq \liminf _{n \rightarrow \infty} \rho\left(x_{n}\right)$ whenever the sequence $\left\{x_{n}\right\}$ is $\rho$-convergent to $x$ in the modular space $X_{\rho}$.

\section{Solution of EquAtion 1.6}

In this section, we find out the general solution of (1.6). Here, we consider the following mixed additive, quadratic and cubic functional equation

$$
\begin{aligned}
& f(n x+y)+f(n x-y) \\
& \quad=n\{f(x+y)+f(x-y)\}+2 f(n x)-2 n f(x)-(1-n)[f(y)-f(-y)]
\end{aligned}
$$

for the fixed integer $n$ such that $n \neq 0, \pm 1$. It is easy to check that the equation (3.1) is a generalized form of 1.4 and that $f(x)=a x^{3}+b x^{2}+c x$ is a solution of it.

Lemma 3.1. Let $X$ and $Y$ be real vector spaces. If the mapping $f: X \rightarrow Y$ satisfies the functional equations (1.5) and (3.1), then $f$ satisfies (1.6). 
Proof. Replacing $x$ by $n x$ in 1.5 , we have

$$
\begin{aligned}
& f(n x+m y)+f(n x-m y) \\
& \quad=m^{2}\{f(n x+y)+f(n x-y)\}+2\left(1-m^{2}\right) f(n x)
\end{aligned}
$$

for fixed integer $m$ with $m \neq 0, \pm 1$. It follows from $(3.2)$ and our assumption that

$$
\begin{aligned}
& f(n x+m y)+f(n x-m y) \\
& =m^{2}[n(f(x+y)+f(x-y))-2 n f(x)+2 f(n x)+(1-n)(f(y)+f(-y))] \\
& \quad+2\left(1-m^{2}\right) f(n x)
\end{aligned}
$$

for all $x, y \in X$. Changing $(m, n)$ by $(n, m)$ in $(3.3)$, we get

$$
\begin{aligned}
& f(m x+n y)+f(m x-n y) \\
& =n^{2}[m(f(x+y)+f(x-y))-2 m f(x)+2 f(m x)+(1-m)(f(y)+f(-y))] \\
& \quad+2\left(1-n^{2}\right) f(m x)
\end{aligned}
$$

for all $x, y \in X$. Adding both sides of 3.3 and 3.4 , we obtain (1.6).

In the sequel, by "the mapping $f: X \rightarrow Y$ satisfies the functional equation (1.6)", we mean that $f$ satisfies 1.6 for the fixed integer $n$ and any integer $m$ such that $m, n \neq 0, \pm 1$ and $m+n \neq 0$.

Proposition 3.2. Let $X$ and $Y$ be real vector spaces. Suppose that the mapping $f: X \rightarrow Y$ satisfies the functional equation (1.6.

(i) If $f$ is an even mapping, then it is quadratic;

(ii) If $f$ is an odd mapping, then the mappings $g, h: X \rightarrow Y$ defined by $g(x):=$ $f(2 x)-8 f(x)$ and $h(x):=f(2 x)-2 f(x)$ are additive and cubic, respectively.

Proof. (i) By our assumption, the equation (1.6 can be rewritten as follows:

$$
\begin{aligned}
f(m x+n y)+f( & m x-n y)+f(n x+m y)+f(n x-m y) \\
= & m n(m+n)[f(x+y)+f(x-y)-2 f(x)-2 f(y)] \\
& +2[f(m x)+f(n x)]+2\left(m^{2}+n^{2}\right) f(y) .
\end{aligned}
$$

Note that $f(0)=0$. Replacing $(x, y, m)$ by $(0, x, n)$ in $(3.5)$, we get

$$
f(n x)=n^{2} f(x)
$$

for all $x \in X$. Putting $m=n$ in (3.5) and using (3.6), we have

$$
(n-1)[f(x+y)+f(x-y)-2 f(x)-2 f(y)]=0
$$

for all $x, y \in X$. Since $n \neq 1$, it follows from (3.7) that $f(x+y)+f(x-y)=$ $2 f(x)+2 f(y)$ for all $x, y \in X$. This means that $f$ is a quadratic mapping.

(ii) The hypothesis shows that the equation $(1.6)$ is equivalent to

$$
\begin{aligned}
& f(m x+n y)+f(m x-n y)+f(n x+m y)+f(n x-m y) \\
& \quad=m n(m+n)[f(x+y)+f(x-y)-2 f(x)]+2[f(m x)+f(n x)] .
\end{aligned}
$$


Letting $y=x$ and $m=2 n$ in 3.8 and using the oddness of $f$, we get

$$
f(3 n x)=3 n^{3}[f(2 x)-2 f(x)]+f(2 n x)+f(n x)
$$

for all $x \in X$ (and for the rest of this proof, all the equations are valid for all $x \in X)$. Putting $y=x$ and $m=3 n$ in (3.8), we obtain

$$
f(4 n x)=6 n^{3}[f(2 x)-2 f(x)]+f(3 n x)+f(n x) .
$$

Replacing $(x, y, m)$ by $(2 x, x, n)$ in $(3.8$, we have

$$
f(3 n x)+f(n x)=n^{3}[f(3 x)+f(x)-2 f(2 x)]+2 f(2 n x) .
$$

Substituting $(x, y, m)$ by $(3 x, x, n)$ in $(3.8)$, we deduce that

$$
f(4 n x)+f(2 n x)=n^{3}[f(4 x)+f(2 x)-2 f(3 x)]+2 f(3 n x) .
$$

Changing $(x, y, m)$ into $(x, 2 x, n)$ in $(3.8)$, we obtain

$$
f(3 n x)-f(n x)=n^{3}[f(3 x)-3 f(x)]+2 f(n x) .
$$

It follows from (3.11) and (3.13) that

$$
f(3 n x)=n^{3}[f(3 x)-f(2 x)-f(x)]+f(2 n x)+f(n x) .
$$

Since $n \neq 0$, the equalities 3.9 and 3.14 imply that

$$
4 f(2 x)-5 f(x)-f(3 x)=0 .
$$

Changing $(x, y, m)$ by $(x, 3 x, n)$ in 3.8 , we get

$$
f(4 n x)-f(2 n x)=n^{3}[f(4 x)-f(2 x)-2 f(x)]+2 f(n x) .
$$

Adding (3.12) to (3.16), we have

$$
f(4 n x)=n^{3}[f(4 x)-f(3 x)-f(x)]+f(3 n x)+f(n x) .
$$

From the relations 3.10 and 3.17$)$, we arrive at

$$
6 f(2 x)-11 f(x)-f(4 x)+f(3 x)=0 .
$$

Plugging 3.15 into 3.18, we find

$$
f(4 x)-10 f(2 x)+16 f(x)=0 .
$$

From the relation 3.19, the desired results can be obtained.

Here and subsequently, given $f: X \rightarrow Y$, for simplicity, we define the difference operator $\Lambda_{m, n} f: X \times X \rightarrow Y$ by

$$
\begin{aligned}
\Lambda_{m, n} f(x, y):= & f(m x+n y)+f(m x-n y)+f(n x+m y)+f(n x-m y) \\
= & m n(m+n)[f(x+y)+f(x-y)-2 f(x)-f(y)-f(-y)] \\
& +2[f(m x)+f(n x)]+\left(m^{2}+n^{2}\right)[f(y)+f(-y)]
\end{aligned}
$$

for all $x, y \in X$, the fixed integer $n$ and any integer $m$ such that $m \neq 0, \pm 1$, $m+n \neq 0$. 


\section{Stability of Equation (1.6) in modular spaces}

In this section, we prove the generalized Hyers-Ulam-Rassias stability of the mixed type additive-quadratic-cubic functional equation 1.6. From now on, we assume that $V$ is a real vector space and $X_{\rho}$ is a complete modular space that satisfies the $\Delta_{2}$-condition and has the Fatou property unless otherwise stated explicitly. In the next theorem we prove the stability of the functional equation (1.6) as a quadratic functional equation (the even case of (1.6) ) in the modular spaces.

Theorem 4.1. Let $s \in\{1,-1\}$. Let $\phi: V \times V \rightarrow[0, \infty)$ be a function such that

$$
\sum_{j=\frac{1}{2}|s-1|}^{\infty} \frac{\kappa^{2|s-1| j}}{4^{j}} \phi\left(2^{s j} x, 2^{s j} y\right)<\infty
$$

for all $x, y \in V$. Suppose that $f: V \rightarrow X_{\rho}$ is an even mapping satisfying $f(0)=0$ (when $s=1$ ) and the inequality

$$
\rho\left(\Lambda_{m, n} f(x, y)\right) \leq \phi(x, y)
$$

for all $x, y \in V$. Then, there exists a unique quadratic mapping $\mathcal{Q}: V \rightarrow X_{\rho}$ such that

$$
\rho(f(x)-\mathcal{Q}(x)) \leq \frac{1}{4} \sum_{j=\frac{1}{2}|s-1|}^{\infty} \frac{\kappa^{2|s-1| j} \Phi\left(2^{j s} x\right)}{4^{j}}
$$

for all $x \in V$, where

$$
\Phi(x)=\frac{1}{2 n^{2}|n-1|}\left[\phi(0, x)+\phi(x, x)+\frac{1}{2} \phi(0,2 x)\right] .
$$

Proof. We first consider $s=1$. Note that in this case $f(0)=0$ is assumed. Replacing $(x, y, m)$ by $(0, x, n)$ in 4.2 , we get

$$
\rho\left(4 f(n x)-4 n^{2} f(x)\right) \leq \phi(0, x)
$$

for all $x \in V$. Substituting $(x, y, m)$ by $(x, x, n)$ in 4.2 , we obtain

$$
\rho\left(2 f(2 n x)-2 n^{3}(f(2 x)-4 f(x))-4 f(n x)-4 n^{2} f(x)\right) \leq \phi(x, x)
$$

for all $x \in V$. The relation 4.6 can be modified as follows:

$$
\begin{aligned}
\rho\left(2 f(2 n x)-2 n^{2} f(2 x)-2 n^{2}(n-1)[f(2 x)\right. & -4 f(x)] \\
& \left.-\left[4 f(n x)-4 n^{2} f(x)\right]\right) \leq \phi(x, x)
\end{aligned}
$$

for all $x \in V$. The relations 4.5 and 4.7 imply that

$$
\rho\left(f(2 n x)-n^{2} f(2 x)-n^{2}(n-1)[f(2 x)-4 f(x)]\right) \leq \frac{1}{2}[\phi(0, x)+\phi(x, x)]
$$

for all $x \in V$. Also, it follows from 4.5 that

$$
\rho\left(f(2 n x)-n^{2} f(2 x)\right) \leq \frac{1}{4} \phi(0,2 x)
$$


for all $x \in V$. Plugging (4.8) into 4.9, we find

$$
\rho\left(\frac{n^{2}(n-1)}{2}[f(2 x)-4 f(x)]\right) \leq \frac{1}{4}[\phi(0, x)+\phi(x, x)]+\frac{1}{8} \phi(0,2 x)
$$

for all $x \in V$. So,

$$
\rho(f(2 x)-4 f(x)) \leq \Phi(x)
$$

for all $x \in V$, where $\Phi(x)$ is defined in 4.4. The inequality 4.10 implies that

$$
\rho\left(f(x)-\frac{f(2 x)}{4}\right) \leq \frac{1}{4} \Phi(x)
$$

for all $x \in V$. Once more, by induction on $k$, one can prove the following functional inequality:

$$
\rho\left(f(x)-\frac{f\left(2^{k} x\right)}{4^{k}}\right) \leq \frac{1}{4} \sum_{j=0}^{k-1} \frac{\Phi\left(2^{j} x\right)}{4^{j}}
$$

for all $x \in V$. Now, changing $x$ by $2^{l} x$ in 4.11, we have

$$
\rho\left(\frac{f\left(2^{l} x\right)}{4^{l}}-\frac{f\left(2^{k+l} x\right)}{4^{k+l}}\right) \leq \frac{1}{4} \sum_{j=l}^{k+l-1} \frac{\Phi\left(2^{j} x\right)}{4^{j}}
$$

for all $x \in V$. Since the right-hand side of the above inequality tends to zero as $l$ goes to infinity, the sequence $\left\{\frac{f\left(2^{k} x\right)}{4^{k}}\right\}$ is a $\rho$-Cauchy sequence in $X_{\rho}$ and so the mentioned sequence is $\rho$-convergent on $X_{\rho}$. Thus, we may define the mapping $\mathcal{Q}: V \rightarrow X_{\rho}$ via $\mathcal{Q}(x)=\rho-\lim _{k \rightarrow \infty} \frac{f\left(2^{k} x\right)}{4^{k}}$ for all $x \in V$. In other words, $\lim _{k \rightarrow \infty} \rho\left(\frac{f\left(2^{k} x\right)}{4^{k}}-\mathcal{Q}(x)\right)=0$. Replacing $(x, y)$ by $\left(2^{k} x, 2^{k} y\right)$ in 4.2 , and dividing the resulting inequality by $4^{k}$, we get

$$
\rho\left(\frac{1}{4^{k}} \Lambda_{m, n} f\left(2^{k} x, 2^{k} y\right)\right) \leq \frac{1}{4^{k}} \rho\left(\Lambda_{m, n} f\left(2^{k} x, 2^{k} y\right)\right) \leq \frac{1}{4^{k}} \phi\left(2^{k} x, 2^{k} y\right)
$$

for all $x, y \in V$. For the fixed and arbitrary integer $m \neq 0, \pm 1$, put $t=9+$ $6|m n(m+n)|+2\left(m^{2}+n^{2}\right)$. By the property $\rho(\alpha t) \leq \alpha \rho(t)$ when $0<\alpha \leq 1$, we 
have

$$
\begin{aligned}
\rho & \left(\frac{1}{t} \Lambda_{m, n} \mathcal{Q}(x, y)\right) \\
= & \rho\left(\frac{1}{t}\left[\Lambda_{m, n} \mathcal{Q}(x, y)-\frac{\Lambda_{m, n} f\left(2^{k} x, 2^{k} y\right)}{4^{k}}+\frac{\Lambda_{m, n} f\left(2^{k} x, 2^{k} y\right)}{4^{k}}\right]\right) \\
\leq & \frac{1}{t} \rho\left(\mathcal{Q}(m x+n y)-\frac{f\left(2^{k}(m x+n y)\right)}{4^{k}}\right)+\frac{1}{t} \rho\left(\mathcal{Q}(m x-n y)-\frac{f\left(2^{k}(m x-n y)\right)}{4^{k}}\right) \\
& +\frac{1}{t} \rho\left(\mathcal{Q}(n x+m y)-\frac{f\left(2^{k}(n x+m y)\right)}{4^{k}}\right)+\frac{1}{t} \rho\left(\mathcal{Q}(n x-m y)-\frac{f\left(2^{k}(n x-m y)\right)}{4^{k}}\right) \\
& +\frac{|m n(m+n)|}{t} \rho\left(\mathcal{Q}(x+y)-\frac{f\left(2^{k}(x+y)\right)}{4^{k}}\right) \\
& +\frac{|m n(m+n)|}{t} \rho\left(\mathcal{Q}(x-y)-\frac{f\left(2^{k}(x-y)\right)}{4^{k}}\right) \\
& +\frac{2|m n(m+n)|}{t} \rho\left(\mathcal{Q}(x)-\frac{f\left(2^{k} x\right)}{4^{k}}\right)+\frac{|2 m n(m+n)|}{t} \rho\left(\mathcal{Q}(y)-\frac{f\left(2^{k} y\right)}{4^{k}}\right) \\
& +\frac{2}{t} \rho\left(\mathcal{Q}(m x)-\frac{f\left(2^{k} m x\right)}{4^{k}}\right)+\frac{2}{t} \rho\left(\mathcal{Q}(n x)-\frac{f\left(2^{k} n x\right)}{4^{k}}\right) \\
& +\frac{2\left(m^{2}+n^{2}\right)}{t} \rho\left(\mathcal{Q}(y)-\frac{f\left(2^{k} y\right)}{4^{k}}\right)+\frac{1}{t} \rho\left(\frac{\Lambda_{m, n} f\left(2^{k} x, 2^{k} y\right)}{4^{k}}\right)
\end{aligned}
$$

for all $x, y \in V$ and all positive integers $k$. Taking the limit as $k \rightarrow \infty$, we see that $\rho\left(\frac{1}{t} \Lambda_{m, n} \mathcal{Q}(x, y)\right)=0$, and hence $\Lambda_{m, n} \mathcal{Q}(x, y)=0$ for all $x, y \in V$. This means that $\mathcal{Q}$ is a quadratic mapping. Now, it follows from the Fatou property of modular $\rho$ that

$$
\rho(f(x)-\mathcal{Q}(x)) \leq \liminf _{\rho \rightarrow \infty}\left(f(x)-\frac{f\left(2^{k} x\right)}{4^{k}}\right) \leq \frac{1}{4} \sum_{j=0}^{k-1} \frac{\Phi\left(2^{j} x\right)}{4^{j}}
$$

for all $x \in V$, which shows that the relation 4.3 holds. For the uniqueness of $\mathcal{Q}$, we assume that there exists another quadratic mapping $\mathcal{Q}_{0}: V \rightarrow X_{\rho}$ such that

$$
\rho\left(f(x)-\mathcal{Q}_{0}(x)\right) \leq \frac{1}{4} \sum_{j=0}^{\infty} \frac{\Phi\left(2^{j} x\right)}{4^{j}}
$$

for all $x \in V$ such that $\mathcal{Q}_{0}\left(x_{*}\right) \neq \mathcal{Q}\left(x_{*}\right)$ for some $x_{*} \in V$. In other words, there is a positive constant $\delta>0$ such that $\rho\left(\mathcal{Q}_{0}\left(x_{*}\right)-\mathcal{Q}\left(x_{*}\right)\right)>\delta$. On the other hand, there is a positive integer $p_{0} \in \mathbb{N}$ such that $\frac{1}{4} \sum_{j=p_{0}}^{\infty} \frac{\Phi\left(2^{j} x\right)}{4^{j}}<\delta$. Since $\mathcal{Q}$ and $\mathcal{Q}_{0}$ are quadratic mappings, we have $\mathcal{Q}_{0}\left(2^{p_{0}} x\right)=4^{p_{0}} \mathcal{Q}_{0}(x)$ and $\mathcal{Q}\left(2^{p_{0}} x\right)=4^{p_{0}} \mathcal{Q}(x)$. 
So,

$$
\begin{aligned}
\delta<\rho\left(\mathcal{Q}_{0}(x)-\mathcal{Q}(x)\right) & \leq \frac{1}{4^{p_{0}}} \rho\left(\mathcal{Q}_{0}\left(2^{p_{0}} x\right)-f\left(2^{p_{0}} x\right)\right)+\frac{1}{4^{p_{0}}} \rho\left(f\left(2^{p_{0}} x\right)-\mathcal{Q}\left(2^{p_{0}} x\right)\right) \\
& \leq \frac{1}{4} \sum_{j=0}^{\infty} \frac{\Phi\left(2^{j+p_{0}} x\right)}{4^{j+p_{0}}}=\frac{1}{4} \sum_{j=p_{0}}^{\infty} \frac{\Phi\left(2^{j} x\right)}{4^{j}}<\delta,
\end{aligned}
$$

which is a contradiction. Now, assume that $s=-1$. Since $\kappa \geq 2$, 4.1 implies that $f(0)=0$. It follows from 4.10 that

$$
\rho\left(f(x)-4 f\left(\frac{x}{2}\right)\right) \leq \Phi\left(\frac{x}{2}\right)
$$

for all $x \in V$. By the convexity of the modular $\rho, \Delta_{2}$-condition and 4.12 , we have

$$
\begin{aligned}
\rho\left(f(x)-4^{2} f\left(\frac{x}{2^{2}}\right)\right) & \leq \frac{1}{4} \rho\left(4 f(x)-4^{2} f\left(\frac{x}{2}\right)\right)+\frac{1}{4} \rho\left(4^{2} f\left(\frac{x}{2}\right)-4^{3} f\left(\frac{x}{2^{2}}\right)\right) \\
& \leq \frac{\kappa^{2}}{4} \Phi\left(\frac{x}{2}\right)+\frac{\kappa^{4}}{4} \Phi\left(\frac{x}{2^{2}}\right)
\end{aligned}
$$

for all $x \in V$. It is routine to show by induction on $k>1$ that

$$
\rho\left(f(x)-4^{k} f\left(\frac{x}{2^{k}}\right)\right) \leq \sum_{j=1}^{k-1} \frac{\kappa^{2(2 j-1)}}{4^{j}} \Phi\left(\frac{x}{2^{j}}\right)+\frac{\kappa^{4(k-1)}}{4^{k-1}} \Phi\left(\frac{x}{2^{k}}\right)
$$

for all $x \in V$. Replacing $x$ by $\frac{x}{2^{l}}$ in 4.13, we get

$$
\begin{gathered}
\rho\left(4^{l} f\left(\frac{x}{2^{l}}\right)-4^{k+l} f\left(\frac{x}{2^{k+l}}\right)\right) \leq \kappa^{2 l} \rho\left(f\left(\frac{x}{2^{l}}\right)-4^{k} f\left(\frac{x}{2^{k+l}}\right)\right) \\
\leq \kappa^{2 l} \sum_{j=1}^{k-1} \frac{\kappa^{2(2 j-1)}}{4^{j}} \Phi\left(\frac{x}{2^{j+l}}\right)+\kappa^{2 l} \frac{\kappa^{4(k-1)}}{4^{k-1}} \Phi\left(\frac{x}{2^{k+l}}\right) \\
\leq \frac{4^{l}}{\kappa^{2 l}} \sum_{j=1+1}^{k+l-1} \frac{\kappa^{2(2 j-1)}}{4^{j}} \Phi\left(\frac{x}{2^{j}}\right)+\frac{4^{l}}{\kappa^{2 l}} \frac{\kappa^{4(k+l-1)}}{4^{k+l-1}} \Phi\left(\frac{x}{2^{k+l}}\right)
\end{gathered}
$$

for all $x \in V$. It follows from (4.1) and 4.14 that the sequence $\left\{4^{k} f\left(\frac{x}{2^{k}}\right)\right\}$ is a $\rho$-Cauchy sequence in $X_{\rho}$. So, there exists a mapping $\mathcal{Q}: V \rightarrow X_{\rho}$ such that $\mathcal{Q}(x)=\rho-\lim _{k \rightarrow \infty} 4^{k} f\left(\frac{x}{2^{k}}\right)$. This means that the mentioned sequence is $\rho$ convergent to $\mathcal{Q}(x)$. Using the $\Delta_{2}$-condition without applying the Fatou property, we obtain

$$
\begin{aligned}
\rho(f(x)-\mathcal{Q}(x)) \leq & \frac{1}{4} \rho\left(4 f(x)-4^{k+1} f\left(\frac{x}{2^{k}}\right)\right)+\frac{1}{4} \rho\left(4^{k+1} f\left(\frac{x}{2^{k}}\right)-4 \mathcal{Q}(x)\right) \\
\leq & \frac{\kappa^{2}}{4} \rho\left(f(x)-4^{k} f\left(\frac{x}{2^{k}}\right)\right)+\frac{\kappa^{2}}{4} \rho\left(4^{k} f\left(\frac{x}{2^{k}}\right)-\mathcal{Q}(x)\right) \\
\leq & \frac{\kappa^{2}}{4} \sum_{j=1}^{k-1} \frac{\kappa^{2(2 j-1)}}{4^{j}} \Phi\left(\frac{x}{2^{j}}\right)+\frac{\kappa^{2}}{4} \frac{\kappa^{4(k-1)}}{4^{k-1}} \Phi\left(\frac{x}{2^{k}}\right) \\
& +\frac{\kappa^{2}}{4} \rho\left(4^{k} f\left(\frac{x}{2^{k}}\right)-\mathcal{Q}(x)\right)
\end{aligned}
$$


for all $x \in V$. Letting $k \rightarrow \infty$, we see that (4.3) holds. The rest of the proof is similar to the case $s=1$. This completes the proof.

The following corollaries are the direct consequences of Theorem 4.1 concerning the stability of $(1.6)$.

Corollary 4.2. Let $V$ be a normed space and $X_{\rho}$ a $\rho$-complete convex modular space. Assume $\theta>0$ and $r>0$ with $r \neq 2, \log _{2}^{\frac{\kappa^{4}}{4}}$. If $f: V \rightarrow X_{\rho}$ is an even mapping satisfying

$$
\rho\left(\Lambda_{m, n} f(x, y)\right) \leq \theta\left(\|x\|^{r}+\|y\|^{r}\right)
$$

for all $x, y \in V$, then there exists a unique quadratic mapping $\mathcal{Q}: V \rightarrow X_{\rho}$ such that

$$
\rho(f(x)-\mathcal{Q}(x)) \leq \begin{cases}\frac{\theta\left(3+2^{r-1}\right)}{2 n^{2}|n-1|\left(4-2^{r}\right)}\|x\|^{r} & r \in(0,2), \\ \frac{\theta\left(3+2^{r-1}\right) \kappa^{4}}{8 n^{2}|n-1|\left(2^{r+2}-\kappa^{4}\right)}\|x\|^{r} & r \in\left(\log _{2}^{\frac{\kappa^{4}}{4}}, \infty\right),\end{cases}
$$

for all $x \in V$.

Proof. We first note that $f(0)=0$. Putting $\phi(x, y)=\theta\left(\|x\|^{r}+\|y\|^{r}\right)$ in Theorem 4.1. one can obtain the first and second inequalities for $s=1$ and $s=-1$, respectively.

Corollary 4.3. Let $V$ be a normed space and $X_{\rho}$ a $\rho$-complete convex modular space. Assume $\theta, p, q>0$ and $r=p+q$ with $r \neq 2, \log _{2}^{\frac{\kappa^{4}}{4}}$. If $f: V \rightarrow X_{\rho}$ is an even mapping satisfying

$$
\rho\left(\Lambda_{m, n} f(x, y)\right) \leq \theta\|x\|^{p}\|y\|^{q}
$$

for all $x, y \in V$, then there exists a unique quadratic mapping $\mathcal{Q}: V \rightarrow X_{\rho}$ such that

$$
\rho(f(x)-\mathcal{Q}(x)) \leq \begin{cases}\frac{\theta}{2 n^{2}|n-1|\left(4-2^{r}\right)}\|x\|^{r} & r \in(0,2), \\ \frac{\theta \kappa^{4}}{8 n^{2}|n-1|\left(2^{r+2}-\kappa^{4}\right)}\|x\|^{r} & r \in\left(\log _{2}^{\frac{\kappa^{4}}{4}}, \infty\right),\end{cases}
$$

for all $x \in V$.

Proof. Refer to the proof of Corollary 4.2

Remark 4.4. We should remember that

(i) in the case $s=1$ of Theorem 4.1, we have used the Fatou property while the $\Delta_{2}$-condition is not applied and vice versa for the case $s=-1$;

(ii) in Corollary 4.2 and Corollary 4.3 if the $\Delta_{2}$-constant is $\kappa=2$, then $\log _{2}^{\frac{\kappa^{4}}{4}}=2$. So, the second conditions convert to $r \in(2, \infty)$.

The idea of the following example is taken from [11. We show the hypothesis $r \neq 2$ can not be removed in Corollary 4.2 
Example 4.5. Let $\theta>0$. For the fixed and arbitrary integers $m, n$ with $m, n \neq$ $0, \pm 1$ and $m+n \neq 0$, set $\mu=\frac{3 \theta}{64 \nu t^{2}}$, where $t=\max \{|m|,|n|\}$ and $\nu=\mid 6 m n(m+$ $n)+2\left(m^{2}+n^{2}\right)+8 \mid$. Consider the function $\psi: \mathbb{R} \rightarrow \mathbb{R}$ defined by

$$
\psi(x)= \begin{cases}\mu x^{2} & |x|<1 \\ \mu & |x| \geq 1\end{cases}
$$

Suppose that the function $f: \mathbb{R} \rightarrow \mathbb{R}$ is defined through

$$
f(x)=\sum_{k=0}^{\infty} \frac{\psi\left(2^{k} x\right)}{4^{k}}, \quad x \in \mathbb{R} .
$$

We have $f(0)=0$ and $f$ is an even function. Furthermore, $\psi$ is continuous and bounded by $\mu$. Since $f$ is a uniformly convergent series of continuous functions, it is continuous and bounded. Indeed, for each $x \in \mathbb{R}$, we have $|f(x)| \leq \frac{4}{3} \mu$. We wish to show that

$$
\left|\Lambda_{m, n} f(x, y)\right| \leq \theta\left(x^{2}+y^{2}\right)
$$

for all $x, y \in \mathbb{R}$. Obviously, 4.15 holds for $x=y=0$. Assume that $x, y \in \mathbb{R}$ with $x^{2}+y^{2}<\frac{1}{16 t^{2}}$. Thus, there exists a positive integer $N$ such that

$$
\frac{1}{2^{2 N+3} t^{2}}<x^{2}+y^{2}<\frac{1}{2^{2 N+2} t^{2}} .
$$

So, $|x|<\frac{1}{4 t}$ and $|y|<\frac{1}{4 t}$, and hence $|x|+|y|<\frac{1}{2 t}$. Therefore,

$$
\begin{aligned}
& \left\{\left|2^{N-1}(m x \pm n y)\right|<1,\left|2^{N-1}(n x \pm m y)\right|<1,\left|2^{N-1} m x\right|<1,\right. \\
& \left|2^{N-1} n x\right|<1,\left|2^{N-1} m y\right|<1,\left|2^{N-1} n y\right|<1,\left|2^{N-1}(x \pm y)\right|<1, \\
& \left.\left|2^{N-1} 2 x\right|<1,\left|2^{N-1} 2 y\right|<1\right\} \subseteq(-1,1) .
\end{aligned}
$$

Since $\psi$ is quadratic on $(-1,1)$, by Proposition $3.2\left|\Lambda_{m, n} \psi\left(2^{k} x, 2^{k} y\right)\right|=0$ for all $k \in\{0,1,2, \ldots, N-1\}$. The last equality and the relation 4.16 imply that

$$
\begin{aligned}
\frac{\left|\Lambda_{m, n} f\left(2^{k} x, 2^{k} y\right)\right|}{x^{2}+y^{2}} & \leq \sum_{k=N}^{\infty} \frac{\left|\Lambda_{m, n} \psi\left(2^{k} x, 2^{k} y\right)\right|}{4^{k}\left(x^{2}+y^{2}\right)} \\
& \leq \sum_{k=0}^{\infty} \frac{\nu \mu}{4^{k} 4^{N}\left(x^{2}+y^{2}\right)} \leq \sum_{k=0}^{\infty} \frac{8 \nu \mu t^{2}}{4^{k}} \leq \frac{64}{3} \nu \mu t^{2}=\theta
\end{aligned}
$$

for all $x, y \in \mathbb{R}$. If $x^{2}+y^{2} \geq \frac{1}{16 t^{2}}$, then

$$
\frac{\left|\Lambda_{m, n} f\left(2^{n} x, 2^{n} y\right)\right|}{x^{2}+y^{2}} \leq \frac{64}{3} \nu \mu t^{2}=\theta .
$$

Therefore, $f$ satisfies 4.15 for all $x, y \in \mathbb{R}$. Now suppose, contrary to our claim, that there exists a number $b \in[0, \infty)$ and a quadratic function $\mathcal{Q}: \mathbb{R} \rightarrow \mathbb{R}$ such that $|f(x)-\mathcal{Q}(x)|<b x^{2}$ for all $x \in \mathbb{R}$. Hence, there is a constant $c \in \mathbb{R}$ such that $\mathcal{Q}(x)=c x^{2}$ for all $x \in \mathbb{R}$. So

$$
|f(x)| \leq(|c|+b) x^{2}
$$


for all $x \in \mathbb{R}$. On the other hand, consider $p \in \mathbb{N}$ such that $(p+1) \mu>|c|+b$. If $x$ is a real number in $\left(0, \frac{1}{2^{N-1}}\right)$, then $2^{k} x \in(0,1)$ for all $k=0,1, \ldots, N-1$. So, for such $x$, we get

$$
f(x)=\sum_{k=0}^{\infty} \frac{\psi\left(2^{k} x\right)}{4^{k}} \geq \sum_{k=0}^{p} \frac{4^{k} \mu x^{2}}{4^{k}}=(p+1) \mu x^{2}>(|c|+b) x^{2} .
$$

The above relation contradicts 4.17).

We have the next result which is analogous to Theorem 4.1 for the functional equation 1.6 in the odd case.

Theorem 4.6. Let $s \in\{1,-1\}$. Let $\phi: V \times V \rightarrow[0, \infty)$ be a function such that

$$
\sum_{j=\frac{1}{2}|s-1|}^{\infty} \frac{\kappa^{|s-1| j}}{2^{j}} \phi\left(2^{s j} x, 2^{s j} y\right)<\infty
$$

for all $x, y \in V$. Suppose that $f: V \rightarrow X_{\rho}$ is an odd mapping satisfying the inequality

$$
\rho\left(\Lambda_{m, n} f(x, y)\right) \leq \phi(x, y)
$$

for all $x, y \in V$. Then, there exists a unique additive mapping $\mathcal{A}: V \rightarrow X_{\rho}$ such that

for all $x \in V$, where

$$
\rho(f(2 x)-8 f(x)-\mathcal{A}(x)) \leq \frac{1}{2} \sum_{j=\frac{1}{2}|s-1|}^{\infty} \frac{\kappa^{|s-1| j} \Gamma\left(2^{j s} x\right)}{2^{j}}
$$

$$
\Gamma(x)=\frac{1}{|n|^{3}}\left(\phi(x, x)+\frac{1}{4}[\phi(x, 2 x)+\phi(2 x, x)+\phi(x, 3 x)+\phi(3 x, x)]\right) .
$$

Proof. Replacing $(x, y, m)$ by $(2 x, x, n)$ in 4.18, we have

$$
\rho\left(2[f(3 n x)+f(n x)]-2 n^{3}[f(3 x)+f(x)-2 f(2 x)]-4 f(2 n x)\right) \leq \phi(2 x, x)
$$

for all $x \in V$. Changing $(x, y, m)$ into $(x, 2 x, n)$ in 4.18 and using the oddness of $f$, we obtain

$$
\rho\left(2[f(3 n x)-f(n x)]-2 n^{3}[f(3 x)-3 f(x)]-4 f(n x)\right) \leq \phi(x, 2 x)
$$

for all $x \in V$. It follows from 4.20 and 4.21 that

$$
\begin{aligned}
\rho\left(2 f(3 n x)-2 n^{3}[f(3 x)-f(2 x)-f(x)]-2[f(2 n x)\right. & +f(n x)]) \\
& \leq \frac{1}{2}[\phi(x, 2 x)+\phi(2 x, x)]
\end{aligned}
$$

for all $x \in V$. Letting $y=x$ and $m=2 n$ in (4.18) and applying the oddness of $f$, we get

$$
\rho\left(2 f(3 n x)-6 n^{3}[f(2 x)-2 f(x)]-2[f(2 n x)+f(n x)]\right) \leq \phi(x, x)
$$

for all $x \in V$. From the relations 4.22 and 4.23 we arrive at

$$
\rho\left(n^{3}[f(3 x)-4 f(2 x)+5 f(x)]\right) \leq \frac{1}{2} \phi(x, x)+\frac{1}{4}[\phi(x, 2 x)+\phi(2 x, x)]
$$


for all $x \in V$. Substituting $(x, y, m)$ by $(3 x, x, n)$ in 4.18, we deduce that

$$
\rho\left(2[f(4 n x)+f(2 n x)]-2 n^{3}[f(4 x)+f(2 x)-2 f(3 x)]-4 f(3 n x)\right) \leq \phi(3 x, x)
$$

for all $x \in V$. Replacing $(x, y, m)$ by $(x, 3 x, n)$ in 4.18, we find

$$
\rho\left(2[f(4 n x)-f(2 n x)]-2 n^{3}[f(4 x)-f(2 x)-2 f(x)]-4 f(n x)\right) \leq \phi(x, 3 x)
$$

for all $x \in V$. Plugging 4.25 into 4.26), we have

$$
\begin{aligned}
\rho\left(2 f(4 n x)-2 n^{3}[f(4 x)-f(3 x)-f(x)]-2[f(3 n x)\right. & +f(n x)]) \\
& \leq \frac{1}{2}[\phi(x, 3 x)+\phi(3 x, x)]
\end{aligned}
$$

for all $x \in V$. Putting $y=x$ and $m=3 n$ in 4.18, we obtain

$$
\rho\left(2 f(4 n x)-12 n^{3}[f(2 x)-2 f(x)]-2[f(3 n x)+f(n x)] \leq \phi(x, x)\right.
$$

for all $x \in V$. The relations 4.27 and 4.28 imply that

$$
\begin{aligned}
\rho\left(n^{3}[6 f(2 x)-11 f(x)-f(4 x)+f(3 x)]\right) & \\
& \leq \frac{1}{2} \phi(x, x)+\frac{1}{4}[\phi(x, 3 x)+\phi(3 x, x)]
\end{aligned}
$$

for all $x \in V$. One can conclude from 4.24 and that

$$
\begin{aligned}
\rho\left(\frac{n^{3}}{2}[f(4 x)-10 f(2 x)+16 f(x)]\right) \leq & \frac{1}{2} \phi(x, x)+\frac{1}{8}[\phi(x, 2 x)+\phi(2 x, x)] \\
& +\frac{1}{8}[\phi(x, 3 x)+\phi(3 x, x)]
\end{aligned}
$$

for all $x \in V$. From the relation 4.30, we have

$$
\rho(f(4 x)-10 f(2 x)+16 f(x)) \leq \Gamma(x)
$$

for all $x \in V$, where $\Gamma(x)$ is defined in 4.19). We can write the relation 4.31) as

$$
\rho(g(2 x)-2 g(x)) \leq \Gamma(x)
$$

for all $x \in V$, where $g(x)=f(2 x)-8 f(x)$. For the rest of the proof, one can repeat the process in the proof of Theorem 4.1 after relation 4.10 to obtain the desired result.

The following results are some consequences of Theorem 4.6 concerning the stability of (1.6) when $f$ is an odd mapping. Since the proofs are similar to the proofs of Corollaries 4.2 and 4.3 we omit them.

Corollary 4.7. Let $V$ be a normed space and $X_{\rho}$ a $\rho$-complete convex modular space. Assume $\theta>0$ and $r>0$ with $r \neq 1, \log _{2}^{\frac{\kappa^{2}}{2}}$. If $f: V \rightarrow X_{\rho}$ is an odd mapping satisfying

$$
\rho\left(\Lambda_{m, n} f(x, y)\right) \leq \theta\left(\|x\|^{r}+\|y\|^{r}\right)
$$


for all $x, y \in V$, then there exists a unique additive mapping $\mathcal{A}: V \rightarrow X_{\rho}$ such that

$$
\rho(f(2 x)-8 f(x)-\mathcal{A}(x)) \leq \begin{cases}\frac{\theta \mathcal{M}}{|n|^{3}\left(2-2^{r}\right)}\|x\|^{r} & r \in(0,1), \\ \frac{\theta \mathcal{M} \kappa^{2}}{4|n|^{3}\left(2^{r+1}-\kappa^{2}\right)}\|x\|^{r} & r \in\left(\log _{2}^{\frac{\kappa^{2}}{2}}, \infty\right)\end{cases}
$$

for all $x \in V$, where

$$
\mathcal{M}=3+2^{r-1}+\frac{1}{2} 3^{r}
$$

Corollary 4.8. Let $V$ be a normed space and $X_{\rho}$ a $\rho$-complete convex modular space. Assume $\theta, p, q>0$ and $r=p+q$ with $r \neq 1, \log _{2}^{\frac{\kappa^{2}}{2}}$. If $f: V \rightarrow X_{\rho}$ is an odd mapping satisfying

$$
\rho\left(\Lambda_{m, n} f(x, y)\right) \leq \theta\|x\|^{p}\|y\|^{q}
$$

for all $x, y \in V$, then there exists a unique additive mapping $\mathcal{A}: V \rightarrow X_{\rho}$ such that

$$
\rho(f(2 x)-8 f(x)-\mathcal{A}(x)) \leq \begin{cases}\frac{\theta \mathcal{N}}{|n|^{3}\left(2-2^{r}\right)}\|x\|^{r} & r \in(0,1), \\ \frac{\theta \mathcal{N} \kappa^{2}}{2|n|^{3}\left(2^{r+1}-\kappa^{2}\right)}\|x\|^{r} & r \in\left(\log _{2}^{\frac{\kappa^{2}}{2}}, \infty\right)\end{cases}
$$

for all $x \in V$, where

$$
\mathcal{N}=1+\frac{1}{4}\left(2^{p}+2^{q}+3^{p}+3^{q}\right)
$$

Theorem 4.9. Let $s \in\{1,-1\}$. Let $\phi: V \times V \rightarrow[0, \infty)$ be a function such that

$$
\sum_{j=\frac{1}{2}|s-1| 2}^{\infty} \frac{\kappa^{3|s-1| j}}{2^{j}} \phi\left(2^{s j} x, 2^{s j} y\right)<\infty
$$

for all $x, y \in V$. Suppose that $f: V \rightarrow X_{\rho}$ is an odd mapping satisfying the inequality 4.18. Then, there exists a unique cubic mapping $\mathcal{C}: V \rightarrow X_{\rho}$ such that

$$
\rho(f(2 x)-2 f(x)-\mathcal{C}(x)) \leq \frac{1}{8} \sum_{j=\frac{1}{2}|s-1|}^{\infty} \frac{\kappa^{3|s-1| j} \Gamma\left(2^{j s} x\right)}{8^{j}}
$$

for all $x \in V$, where $\Gamma(x)$ is defined in 4.19).

Proof. It follows from the proof of Theorem 4.6 and specially from the relation 4.31 that

$$
\rho(h(2 x)-8 h(x)) \leq \Gamma(x)
$$

for all $x \in V$, where $h(x)=f(2 x)-2 f(x)$ and $\Gamma(x)$ is defined in 4.19). For the rest of the proof, one can refer to the proofs of Theorems 4.1 and 4.6

Corollary 4.10. Let $V$ be a normed space and $X_{\rho}$ a $\rho$-complete convex modular space. Assume $\theta>0$ and $r \in \mathbb{R}$ with $r \neq 3, \log _{2}^{\frac{\kappa^{6}}{8}}$. If $f: V \rightarrow X_{\rho}$ is an odd mapping satisfying

$$
\rho\left(\Lambda_{m, n} f(x, y)\right) \leq \theta\left(\|x\|^{r}+\|y\|^{r}\right)
$$


for all $x, y \in V$, then there exists a unique cubic mapping $\mathcal{C}: V \rightarrow X_{\rho}$ such that

$$
\rho(f(2 x)-2 f(x)-\mathcal{C}(x)) \leq \begin{cases}\frac{\theta \mathcal{M}}{|n|^{3}\left(8-2^{r}\right)}\|x\|^{r} & r \in(0,3), \\ \frac{\theta \mathcal{M} \kappa^{6}}{8|n|^{3}\left(2^{r+3}-\kappa^{6}\right)}\|x\|^{r} & r \in\left(\log _{2}^{\frac{\kappa^{6}}{8}}, \infty\right)\end{cases}
$$

for all $x \in V$, where $\mathcal{M}$ is defined in 4.32 .

Corollary 4.11. Let $V$ be a normed space and $X_{\rho}$ a $\rho$-complete convex modular space. Assume $\theta, p, q>0$ and $r=p+q$ with $r \neq 3, \log _{2}^{\frac{\kappa^{6}}{8}}$. If $f: V \rightarrow X_{\rho}$ is an odd mapping satisfying

$$
\rho\left(\Lambda_{m, n} f(x, y)\right) \leq \theta\|x\|^{p}\|y\|^{q}
$$

for all $x, y \in V$, then there exists a unique cubic mapping $\mathcal{C}: V \rightarrow X_{\rho}$ such that

$$
\rho(f(2 x)-2 f(x)-\mathcal{C}(x)) \leq \begin{cases}\frac{\theta \mathcal{N}}{|n|^{3}\left(8-2^{r}\right)}\|x\|^{r} & r \in(0,3), \\ \frac{\theta \mathcal{N} \kappa^{6}}{8|n|^{3}\left(2^{r+3}-\kappa^{6}\right)}\|x\|^{r} & r \in\left(\log _{2}^{\frac{\kappa^{6}}{8}}, \infty\right)\end{cases}
$$

for all $x \in V$, where $\mathcal{N}$ is defined in 4.33.

Here, by using Theorems 4.1 4.6 and 4.9. we prove the generalized HyersUlam-Rassias stability of the mixed type additive, quadratic and cubic functional equation (1.6) when $f$ is an arbitrary mapping.

Theorem 4.12. Let $s \in\{1,-1\}$ and $t \in\{1,2,3\}$. Let $\phi: V \times V \rightarrow[0, \infty)$ be $a$ function such that

$$
\sum_{j=\frac{1}{2}|s-1|}^{\infty} \frac{\kappa^{t|s-1| j}}{2^{t j}} \phi\left(2^{s j} x, 2^{s j} y\right)<\infty
$$

for all $x, y \in V$. Suppose that $f: V \rightarrow X_{\rho}$ is a mapping satisfying $f(0)=0$ (when $s=1$ ) and the inequality

$$
\rho\left(\Lambda_{m, n} f(x, y)\right) \leq \phi(x, y)
$$

for all $x, y \in V$. Then, there exists a unique additive mapping $\mathcal{A}: V \rightarrow X_{\rho}$, a unique quadratic mapping $\mathcal{Q}: V \rightarrow X_{\rho}$, and a unique cubic mapping $\mathcal{C}: V \rightarrow X_{\rho}$ such that

$$
\begin{aligned}
\rho(f(x)- & \mathcal{A}(x)-\mathcal{Q}(x)-\mathcal{C}(x)) \leq \frac{\kappa}{24} \sum_{j=\frac{1}{2}|s-1|}^{\infty} \frac{\kappa^{|s-1| j} \widetilde{\Gamma}\left(2^{j s} x\right)}{2^{j}} \\
& +\frac{\kappa}{8} \sum_{j=\frac{1}{2}|s-1|}^{\infty} \frac{\kappa^{2|s-1| j} \widetilde{\Phi}\left(2^{j s} x\right)}{4^{j}}+\frac{\kappa}{96} \sum_{j=\frac{1}{2}|s-1|}^{\infty} \frac{\kappa^{3|s-1| j} \widetilde{\Gamma}\left(2^{j s} x\right)}{8^{j}}
\end{aligned}
$$

for all $x \in V$, where

$$
\widetilde{\Phi}(x)=\frac{1}{2 n^{2}|n-1|}\left[\Phi(0, x)+\Phi(x, x)+\frac{1}{2} \Phi(0,2 x)\right]
$$


and

$$
\widetilde{\Gamma}(x)=\frac{1}{|n|^{3}}\left(\Phi(x, x)+\frac{1}{4}[\Phi(x, 2 x)+\Phi(2 x, x)+\Phi(x, 3 x)+\Phi(3 x, x)]\right)
$$

in which

$$
\Phi(x, y)=\frac{1}{2}[\phi(x, y)+\phi(-x,-y)]
$$

Proof. To achieve our aim, we decompose $f$ into its even and odd parts by setting

$$
f_{o}(x)=\frac{1}{2}(f(x)-f(-x)), \quad f_{e}(x)=\frac{1}{2}(f(x)+f(-x)), \quad x \in X .
$$

We have $\rho\left(\Lambda_{m, n} f_{o}(x, y)\right) \leq \Phi(x, y)$ and $\rho\left(\Lambda_{m, n} f_{e}(x, y)\right) \leq \Phi(x, y)$ for all $x, y \in V$, where $\Phi(x, y)$ is given in 4.37). It follows from Theorem 4.1 that there exists a unique quadratic mapping $\mathcal{Q}: V \rightarrow X_{\rho}$ such that

$$
\rho\left(f_{e}(x)-\mathcal{Q}(x)\right) \leq \frac{1}{4} \sum_{j=\frac{1}{2}|s-1|}^{\infty} \frac{\kappa^{2|s-1| j} \widetilde{\Phi}\left(2^{j s} x\right)}{4^{j}}
$$

for all $x \in V$, with $\widetilde{\Phi}(x)$ as defined in 4.35 . So

$$
\rho\left(2 f_{e}(x)-2 \mathcal{Q}(x)\right) \leq \frac{\kappa}{4} \sum_{j=\frac{1}{2}|s-1|}^{\infty} \frac{\kappa^{2|s-1| j} \widetilde{\Phi}\left(2^{j s} x\right)}{4^{j}}
$$

for all $x \in V$. Also, Theorems 4.6 and 4.9 imply that there exists a unique additive mapping $\mathcal{A}_{0}: X \rightarrow Y$ and a unique cubic mapping $\mathcal{C}_{0}: X \rightarrow Y$ such that

$$
\rho\left(f_{o}(2 x)-8 f_{o}(x)-\mathcal{A}_{0}(x)\right) \leq \frac{1}{2} \sum_{j=\frac{1}{2}|s-1|}^{\infty} \frac{\kappa^{|s-1| j} \widetilde{\Gamma}\left(2^{j s} x\right)}{2^{j}}
$$

and

$$
\rho\left(f_{o}(2 x)-2 f_{o}(x)-\mathcal{C}_{0}(x)\right) \leq \frac{1}{8} \sum_{j=\frac{1}{2}|s-1|}^{\infty} \frac{\kappa^{3|s-1| j} \widetilde{\Gamma}\left(2^{j s} x\right)}{8^{j}}
$$

for all $x \in V$, where $\widetilde{\Gamma}(x)$ is defined in 4.36). It follows from the inequalities 4.39) and 4.40 that

$$
\begin{aligned}
\rho\left(3 f_{o}(x)+\frac{1}{2} \mathcal{A}_{0}(x)-\frac{1}{2} \mathcal{C}_{0}(x)\right) \leq & \frac{1}{4} \sum_{j=\frac{1}{2}|s-1|}^{\infty} \frac{\kappa^{|s-1| j} \widetilde{\Gamma}\left(2^{j s} x\right)}{2^{j}} \\
& +\frac{1}{16} \sum_{j=\frac{1}{2}|s-1|}^{\infty} \frac{\kappa^{3|s-1| j} \widetilde{\Gamma}\left(2^{j s} x\right)}{8^{j}}
\end{aligned}
$$


for all $x \in V$. The relation 4.41 implies that

$$
\begin{aligned}
\rho\left(2 f_{o}(x)+\frac{1}{3} \mathcal{A}_{0}(x)-\frac{1}{3} \mathcal{C}_{0}(x)\right) \leq & \frac{\kappa}{12} \sum_{j=\frac{1}{2}|s-1|}^{\infty} \frac{\kappa^{|s-1| j} \widetilde{\Gamma}\left(2^{j s} x\right)}{2^{j}} \\
& +\frac{\kappa}{48} \sum_{j=\frac{1}{2}|s-1|}^{\infty} \frac{\kappa^{3|s-1| j} \widetilde{\Gamma}\left(2^{j s} x\right)}{8^{j}}
\end{aligned}
$$

for all $x \in V$. Now, by (4.38) and 4.42 we can obtain the inequality (4.34), where $\mathcal{A}(x)=-\frac{1}{6} \mathcal{A}_{0}(x)$ and $\mathcal{C}(x)=\frac{1}{6} \mathcal{C}_{0}(x)$.

The following corollaries are the direct consequences of Theorem 4.1 concerning the stability of 1.6 .

Corollary 4.13. Let $V$ be a normed space and $X_{\rho}$ a $\rho$-complete convex modular space. Assume $\theta>0$ and $r>0$ with $r \neq t, \log _{2} \frac{\kappa^{2 t}}{2^{t}}$ when $t \in\{1,2,3\}$. If $f: V \rightarrow X_{\rho}$ is a mapping satisfying

$$
\rho\left(\Lambda_{m, n} f(x, y)\right) \leq \theta\left(\|x\|^{r}+\|y\|^{r}\right)
$$

for all $x, y \in V$, then there exist a unique additive mapping $\mathcal{A}: V \rightarrow X_{\rho}$, a unique quadratic mapping $\mathcal{Q}: V \rightarrow X_{\rho}$, and a unique cubic mapping $\mathcal{C}: V \rightarrow X_{\rho}$ such that

$$
\begin{aligned}
& \rho(f(x)-\mathcal{A}(x)-\mathcal{Q}(x)-\mathcal{C}(x)) \\
& \leq\left\{\begin{array}{l}
{\left[\frac{1}{12|n|^{3}\left(2-2^{r}\right)}\|x\|^{r}+\frac{\left(3+2^{r-1}\right)}{4 n^{2}|n-1|\left(4-2^{r}\right)}+\frac{1}{12|n|^{3}\left(8-2^{r}\right)}\right] \theta \mathcal{M} \kappa\|x\|^{r}, \quad 0<r<1,} \\
{\left[\frac{\kappa^{2}}{24|n|^{3}\left(2^{r+1}-\kappa^{2}\right)}+\frac{\left(3+2^{r-1}\right)}{4 n^{2}|n-1|\left(4-2^{r}\right)}+\frac{1}{12|n|^{3}\left(8-2^{r}\right)}\right] \theta \mathcal{M} \kappa\|x\|^{r}, \quad \log _{2}^{\frac{\kappa^{2}}{2}}<r<2,} \\
{\left[\frac{\kappa^{2}}{24|n|^{3}\left(2^{r+1}-\kappa^{2}\right)}+\frac{\left(3+2^{r-1}\right) \kappa^{4}}{16 n^{2}|n-1|\left(2^{r+2}-\kappa^{4}\right)}+\frac{1}{12|n|^{3}\left(8-2^{r}\right)}\right] \theta \mathcal{M} \kappa\|x\|^{r}, \quad \log _{2}^{\frac{\kappa^{4}}{4}}<r<3,} \\
{\left[\frac{\kappa^{2}}{24|n|^{3}\left(2^{r+1}-\kappa^{2}\right)}+\frac{\left(3+2^{r-1}\right) \kappa^{4}}{16 n^{2}|n-1|\left(2^{r+2}-\kappa^{4}\right)}+\frac{\kappa^{6}}{96|n|^{3}\left(2^{r+3}-\kappa^{6}\right)}\right] \theta \mathcal{M} \kappa\|x\|^{r}, \quad r>\log _{2}^{\frac{\kappa^{8}}{8}}}
\end{array}\right.
\end{aligned}
$$

for all $x \in V$, where $\mathcal{M}$ is defined in 4.32 .

Corollary 4.14. Let $V$ be a normed space and $X_{\rho}$ a $\rho$-complete convex modular space. Assume $\theta, p, q>0$ and $r=p+q$ with $r \neq t, \log _{2}{ }^{\frac{\kappa^{2 t}}{2^{t}}}$ when $t \in\{1,2,3\}$. If $f: V \rightarrow X_{\rho}$ is a mapping satisfying

$$
\rho\left(\Lambda_{m, n} f(x, y)\right) \leq \theta\|x\|^{p}\|y\|^{q}
$$

for all $x, y \in V$, then there exist a unique additive mapping $\mathcal{A}: V \rightarrow X_{\rho}$, a unique quadratic mapping $\mathcal{Q}: V \rightarrow X_{\rho}$, and a unique cubic mapping $\mathcal{C}: V \rightarrow X_{\rho}$ such 
that

$$
\begin{aligned}
& \rho(f(x)-\mathcal{A}(x)-\mathcal{Q}(x)-\mathcal{C}(x)) \\
& \leq\left\{\begin{array}{l}
{\left[\frac{1}{12|n|^{3}\left(2-2^{r}\right)}+\frac{1}{4 n^{2}|n-1|\left(4-2^{r}\right)}+\frac{1}{12|n|^{3}\left(8-2^{r}\right)}\right] \theta \mathcal{N} \kappa\|x\|^{r}, \quad 0<r<1,} \\
{\left[\frac{\kappa^{2}}{24|n|^{3}\left(2^{r+1}-\kappa^{2}\right)}+\frac{1}{4 n^{2}|n-1|\left(4-2^{r}\right)}+\frac{1}{12|n|^{3}\left(8-2^{r}\right)}\right] \theta \mathcal{N} \kappa\|x\|^{r}, \quad \log _{2}^{\frac{\kappa^{2}}{2}}<r<2,} \\
{\left[\frac{\kappa^{2}}{24|n|^{3}\left(2^{r+1}-\kappa^{2}\right)}+\frac{\kappa^{4}}{16 n^{2}|n-1|\left(2^{r+2}-\kappa^{4}\right)}+\frac{1}{12|n|^{3}\left(8-2^{r}\right)}\right] \theta \mathcal{N} \kappa\|x\|^{r}, \quad \log _{2}^{\frac{\kappa^{4}}{4}}<r<3,} \\
{\left[\frac{\kappa^{2}}{24|n|^{3}\left(2^{r+1}-\kappa^{2}\right)}+\frac{\kappa^{4}}{16 n^{2}|n-1|\left(2^{r+2}-\kappa^{4}\right)}+\frac{\kappa^{6}}{96|n|^{3}\left(2^{r+3}-\kappa^{6}\right)}\right] \theta \mathcal{N} \kappa\|x\|^{r}, \quad r>\log _{2}^{\frac{\kappa^{8}}{8}}}
\end{array}\right.
\end{aligned}
$$

for all $x \in V$, where $\mathcal{N}$ is defined in 4.33 .

\section{REFERENCES}

[1] T. Aoki, On the stability of the linear transformation in Banach spaces, J. Math. Soc. Japan. 2 (1950), 64-66. MR 0040580

[2] A. Bodaghi, Intuitionistic fuzzy stability of the generalized forms of cubic and quartic functional equations, J. Intell. Fuzzy Syst. 30 (2016), no. 4, 2309-2317.

[3] A. Bodaghi, Stability of a quartic functional equation, Scientific World J. 2014, Art. ID 752146, 9 pages.

[4] A. Bodaghi, Stability of a mixed type additive and quartic function equation, Filomat 28 (2014), no. 8, 1629-1640. MR 3360112

[5] A. Bodaghi, Ulam stability of a cubic functional equation in various spaces, Mathematica 55(78) (2013), no. 2, 125-141. MR 3243160

[6] A. Bodaghi and S. O. Kim, Stability of a functional equation deriving from quadratic and additive functions in non-Archimedean normed spaces, Abstr. Appl. Anal. 2013, Art. ID 198018, 10 pages. MR 3121484

[7] A. Bodaghi, S. M. Moosavi and H. Rahimi, The generalized cubic functional equation and the stability of cubic Jordan *-derivations, Ann. Univ. Ferrara Sez. VII Sci. Mat. 59 (2013), no. 2, 235-250. MR 3120036

[8] S. Czerwik, On the stability of the quadratic mapping in normed spaces, Abh. Math. Sem. Univ. Hamburg 62 (1992), 59-64. MR 1182841.

[9] M. Eshaghi Gordji, Stability of a functional equation deriving from quartic and additive functions, Bull. Korean Math. Soc. 47 (2010), no. 3, 491-502. MR 2666371

[10] M. Eshaghi Gordji, M. Bavand Savadkouhi and Th. M. Rassias, Stability of generalized mixed type additive-quadratic-cubic functional equation in non-Archimedean spaces, preprint, 2009. arXiv:0909.5692 [math.FA].

[11] Z. Gajda, On stability of additive mappings, Internat. J. Math. Math. Sci. 14 (1991), no. 3, 431-434. MR 1110036

[12] D. H. Hyers, On the stability of the linear functional equation, Proc. Nat. Acad. Sci. U.S.A., 27 (1941), no. 4, 222-224. MR 0004076

[13] K. W. Jun and H. M. Kim, The generalized Hyers-Ulam-Rassias stability of a cubic functional equation, J. Math. Anal. Appl. 274 (2002), no. 2, 267-278. MR 1936735

[14] K. W. Jun and H. M. Kim, On the Hyers-Ulam-Rassias stability of a general cubic functional equation, Math. Inequal. Appl. 6 (2003), no. 2, 289-302. MR 1974608 
[15] M. A. Khamsi, Quasicontraction mappings in modular spaces without $\Delta_{2}$-condition, Fixed Point Theory Appl. 2008, Art. ID 916187, 6 pp. MR 2438709

[16] H. M. Kim and Y. S. Hong, Approximate quadratic mappings in modular spaces, Int. J. Pure. Appl. Math. 116 (2017), no. 1, 31-43.

[17] H. M. Kim and H. Y. Shin, Refined stability of additive and quadratic functional equations in modular spaces, J. Inequal. Appl. 2017, Paper no. 146, 13 pp. MR 3665807

[18] H. Koh and D. Kang, On the stability of a generalized cubic functional equation, Bull. Korean Math. Soc. 45 (2008), no. 4, 739-748. MR 2463150

[19] M. Krbec, Modular interpolation spaces I, Z. Anal. Anwendungen 1 (1982), no. 1, 25-40. MR 0690756

[20] S. H. Lee, S. M. Im and I. S. Hwang, Quartic functional equations, J. Math. Anal. Appl. 307 (2005), no. 2, 387-394. MR 2142432

[21] W. A. J. Luxemburg, Banach function spaces, Ph.D. Thesis, Delft University of Technology, Delft, The Netherlands, 1955. MR 0072440

[22] L. Maligranda, Orlicz Spaces and Interpolation. Seminários de Matemática, 5, Universidade Estadual de Campinas, Departamento de Matemática, Campinas, 1989. MR 2264389

[23] S. Mazur and W. Orlicz, On some classes of linear spaces, Studia Math. 17 (1958), 97-119. MR 0098319

[24] J. Musielak and W. Orlicz, On modular spaces, Studia Math. 18 (1959), 49-65. MR 0101487

[25] J. Musielak and W. Orlicz, Some remarks on modular spaces, Bull. Acad. Polon. Sci. Sér. Sci. Math. Astron. Phys. 7 (1959), 661-668. MR 0112017

[26] A. Najati, The generalized Hyers-Ulam-Rassias stability of a cubic functional equation, Turkish J. Math. 31 (2007), no. 4, 395-408. MR 2364909

[27] H. Nakano, Modulared Semi-Ordered Linear Spaces, Maruzen, Tokyo, 1950. MR 0038565

[28] P. Narasimman and A. Bodaghi, Solution and stability of a mixed type functional equation, Filomat 31 (2017), no. 5, 1229-1239. MR 3630041

[29] W. Orlicz, Collected Papers, vols. I, II. PWN, Warszawa, 1988. MR 0963250

[30] C. Park, A. Bodaghi and S. O. Kim, A fixed point approach to stability of additive mappings in modular spaces without $\Delta_{2}$-conditions, J. Comput. Anal. Appl. 24 (2018), no. 6, 10381048. MR 3751931

[31] C. Park, J. M. Rasias, A. Bodaghi and S. O. Kim, Approximate homomorphisms from ternary semigroups to modular spaces, Rev. R. Acad. Cienc. Exactas Fís. Nat. Ser. A Mat. RACSAM 113 (2019), no. 3, 2175-2188. MR 3956242

[32] J. M. Rassias, On approximation of approximately linear mappings by linear mappings, J. Funct. Anal. 46 (1982), no. 1, 126-130. MR 0654469

[33] J. M. Rassias, Solution of the Ulam stability problem for cubic mappings, Glas. Mat. Ser. III 36(56) (2001), no. 1, 63-72. MR 1842825

[34] Th. M. Rassias, On the stability of the linear mapping in Banach spaces, Proc. Amer. Math. Soc. 72 (1978), no. 2, 297-300. MR 0507327

[35] G. Sadeghi, A fixed point approach to stability of functional equations in modular spaces. Bull. Malays. Math. Sci. Soc. 37 (2014), no. 2, 333-344. MR 3188040

[36] F. Skof, Proprietà locali e approssimazione di operatori, Rend. Sem. Mat. Fis. Milano 53 (1983), 113-129. MR 858541

[37] J. Tabor, stability of the Cauchy functional equation in quasi-Banach spaces, Ann. Polon. Math. 83 (2004), no. 3, 243-255. MR 2111711. 
[38] S. M. Ulam, Problems in Modern Mathematics, Science Editions Wiley, New York, 1964. MR 0280310

[39] L. G. Wang, K. P. Xu and Q. W. Liu, On the stability of a mixed functional equation deriving from additve, quadratic and cubic mappings, Acta Math. Sin. (Engl. Ser.) 30 (2014), no. 6, 1033-1049. MR 3200450.

[40] K. Wongkum, P. Chaipunya and P. Kumam, On the generalized Ulam-Hyers-Rassias stability of quadratic mappings in modular spaces without $\Delta_{2}$-conditions. J. Funct. Spaces 2015, Article ID 461719, 6 pp. MR 3352130

[41] T. Z. Xu, J. M. Rassias and W. X. Xu, A generalized mixed quadratic-quartic functional equation, Bull. Malays. Math. Sci. Soc. 35 (2012), no. 3, 633-649. MR 2923655

M. Maghsoudi

Department of Mathematics, South Tehran Branch, Islamic Azad University, Tehran, Iran

mohammadmaghsoudi5@gmail.com

A. Bodaghi ${ }^{\bowtie}$

Young Researchers and Elite Club, Islamshahr Branch, Islamic Azad University, Islamshahr, Iran

abasalt.bodaghi@gmail.com

\author{
A. Niazi Motlagh \\ Department of Mathematics, Faculty of Basic Sciences, University of Bojnord, P.O. Box 1339, \\ Bojnord, Iran \\ a.niazi@ub.ac.ir, niazimotlagh@gmail.com \\ M. Karami \\ Department of Mathematics, South Tehran Branch, Islamic Azad University, Tehran, Iran \\ m-karami@azad.ac.ir
}

Received: November 4, 2018

Accepted: December 27, 2018 